\title{
Numerical approximation of the viscous quantum hydrodynamic model for semiconductors*
}

\author{
Ansgar Jüngel ${ }^{\dagger}$ and Shaoqiang Tang T $^{\ddagger}$
}

\begin{abstract}
The viscous quantum hydrodynamic equations for semiconductors with constant temperature are numerically studied. The model consists of the one-dimensional Euler equations for the electron density and current density, including a quantum correction and viscous terms, coupled to the Poisson equation for the electrostatic potential. The equations can be derived formally from a Wigner-Fokker-Planck model by a moment method. Two different numerical techniques are used: a hyperbolic relaxation scheme and a central finite-difference method. By simulating a ballistic diode and a resonant tunneling diode, it is shown that numerical or physical viscosity changes significantly the behavior of the solutions. Moreover, the current-voltage characteristics show multiple regions of negative differential resistance and hysteresis effects.
\end{abstract}

AMS Classification. 65M06, 76Y05.

Keywords. Quantum hydrodynamics, finite differences, relaxation scheme, resonant tunneling diode.

\section{Introduction}

Quantum semiconductor devices, like high-electron-mobility transistors (HEMT), superlattices or resonant tunneling diodes, are becoming of increasing importance in state-of-theart semiconductor modeling. These devices rely on quantum tunneling of charge carriers

*The first author acknowledges partial support from the Project "Hyperbolic and Kinetic Equations" of the European Union, grant HPRN-CT-2002-00282, and from the Deutsche Forschungsgemeinschaft, grants JU359/3 (Gerhard-Hess Award) and JU359/5 (Priority Program "Multi-scale Problems"). The second author has been supported by the Chinese Special Funds for the Major State Basic Research Project "Nonlinear Science" and NSFC under grant number 100271003.

${ }^{\dagger}$ Fachbereich Mathematik und Informatik, Universität Mainz, Staudingerweg 9, 55099 Mainz, Germany; e-mail: juengel@mathematik.uni-mainz.de.

${ }^{\ddagger}$ LTCS, Department of Mechanics and Engineering Science, Peking University, Beijing 100871, P. R. China; e-mail: maotang@pku.edu.cn. 
through potential barriers for their operation. The fundamental model for quantum devices is the kinetic Wigner equation (or the formally equivalent Schrödinger equation) [31, 37]:

$$
\partial_{t} w+\frac{\hbar}{m} k \cdot \nabla_{x} w+\frac{q}{\hbar} \theta[V](w)=0,
$$

solved in a bounded domain representing the semiconductor device and supplemented with appropriate initial and boundary conditions. Here, $w=w(x, k, t)$ is the Wigner distribution function depending on the space variable $x \in \mathbb{R}^{d}(d \geq 1)$, the wave vector $k \in \mathbb{R}^{d}$, and the time $t>0$. The physical parameters are the reduced Planck constant $\hbar=h / 2 \pi$, the effective mass $m$ of the electrons, and the elementary charge $q$. The operator $\theta[V]$ is defined in the sense of pseudo-differential operators [41] as

$$
\begin{aligned}
(\theta[V])(w)(x, k, t)= & \frac{i}{(2 \pi)^{d}} \int_{\mathbb{R}^{d}} \int_{\mathbb{R}^{d}} \frac{m}{\hbar}\left[V\left(x+\frac{\eta}{2}, t\right)-V\left(x-\frac{\eta}{2}, t\right)\right] \\
& \times w\left(x, k^{\prime}, t\right) e^{-i\left(k-k^{\prime}\right) \cdot \eta} d k^{\prime} d \eta
\end{aligned}
$$

where $V=V(x, t)$ is the electrostatic (mean-feld-type) potential, selfconsistently given by the Poisson equation

$$
\operatorname{div}_{x}\left(\varepsilon_{s} \nabla_{x} V\right)=q(n-C(x)), \quad x \in \mathbb{R}^{d},
$$

with the permittivity $\varepsilon_{s}$ of the semiconductor material and the concentration of fixed charged background ions $C(x)$ (doping profile). The macroscopic particle density $n(x, t)$ and current density $J(x, t)$ are related to the Wigner function by

$$
n(x, t)=\int_{\mathbb{R}^{d}} w(x, k, t) d p, \quad J(x, t)=\frac{q}{m} \int_{\mathbb{R}^{d}} w(x, k, t) p d p,
$$

with the momentum $p=\hbar k$.

Simulations of the Wigner equation are computationally expensive since the Wigner distribution function is a function of time, space, and wave vector. Moreover, unphysical reflections on the domain boundary have to be avoided, which is a delicate problem. Therefore, in recent years, macroscopic descriptions for quantum semiconductor simulations have been investigated. They have the advantages that they are computationally less expensive than its microscopic counterparts, they are expressed in terms of intuitive macroscopic quantities like particle density and current density, and macroscopic (classical) boundary conditions can be imposed. In the following we review some fluid-type quantum models used in the literature.

It is well known since 1927 that there exists a fluiddynamical formulation of the Schrödinger equation [36]. In fact, by separating the real and the complex part of the single-state Schrödinger equation, the electron density $n(x, t)$ and current density $J(x, t)$ are satisfying (formally) the Madelung (or quantum hydrodynamic) equations

$$
\begin{aligned}
\partial_{t} n+\frac{1}{q} \operatorname{div} J & =0, \\
\partial_{t} J+\frac{1}{q} \operatorname{div}\left(\frac{J \otimes J}{n}\right)-\frac{q^{2}}{m} n \nabla V-\frac{q \hbar^{2}}{6 m^{2}} n \nabla\left(\frac{\Delta \sqrt{n}}{\sqrt{n}}\right) & =0,
\end{aligned}
$$


where the symbol $\nabla$ denotes the spatial gradient and $J \otimes J$ the tensor with components $J_{i} J_{k}$. The above model can be interpreted as a dispersive regularization of the pressureless Euler equations [25].

In order to incorporate non-constant temperature effects, a small-temperature ansatz [24] or a mixed state of single-particle Wigner functions [25] can be used, which yields the additional term $\left(q k_{B} T_{0} / m\right) \nabla n$ on the left-hand side of (3) (in the case of a constant scalar particle temperature $T_{0}$ ), where $k_{B}$ denotes the Boltzmann constant. Gardner [20] used a moment method in order to derive formally quantum hydrodynamic equations (also for non-constant temperature). The moment equations are closed taking an approximation of the quantum thermal equilibrium due to Wigner [42]. A quantum hydrodynamic model with a "smooth" potential has been derived in [22, 23]. Related quantum fluid-type models are the quantum moment hydrodynamics systems of [14], the quantum energy-transport models of [13] and the quantum drift-diffusion equations first used in [1] and numerically solved, e.g., in [34, 39].

These approaches do not include collisions of electrons with impurities of the semiconductor or with phonons (except the relaxation-time term of [20]). In fact, a quantum theory of collisions is still at a rather early stage (see, e.g., $[3,6,16,17]$ ). A collision operator of Fokker-Planck-type, which goes back to [7], has been proposed in $[4,8]$. With this operator the Wigner equation becomes

$$
\partial_{t} w+\frac{\hbar}{m} k \cdot \nabla_{x} w+\frac{q}{\hbar} \theta[V](w)=\frac{D_{p p}}{\hbar^{2}} \Delta_{k} w+\frac{1}{\tau_{0}} \operatorname{div}_{k}(k w)+\frac{D_{p q}}{\hbar} \operatorname{div}_{x}\left(\nabla_{k} w\right)+D_{q q} \Delta_{x} w
$$

with constants

$$
D_{p p}=\frac{k_{B} T_{0}}{m \tau_{0}}, \quad D_{p q}=\frac{\Omega \hbar^{2}}{6 \pi k_{B} T_{0} \tau_{0}}, \quad D_{q q}=\frac{\hbar^{2}}{12 m k_{B} T_{0} \tau_{0}},
$$

where $\tau_{0}$ denotes the momentum relaxation time and $\Omega$ the cut-off frequency of the reservoir oscillators. More precisely, this model governs the dynamical evolution of an electron ensemble in the single-particle Hartree approximation interacting dissipatively with an idealized heat bath consisting of an ensemble of harmonic oscillators and modeling the semiconductor lattice. The constants $D_{p p}, D_{p q}$ and $D_{q q}$ constitute the phase-space diffusion matrix of the system, and the term $\operatorname{div}_{k}(k w) / \tau_{0}$ can be interpreted as a friction term. For a discussion of this model we refer to $[4,26]$.

From the Wigner-Fokker-Planck equation (4), moment equations as in [20] can be derived, using an $O\left(\hbar^{2}\right)$ approximation of the quantum thermal equilibrium state as a closure condition. In fact, the only difference is the integration of the Fokker-Planck term in the wave vector space. The resulting equations are as follows (see the appendix of [26] for details):

$$
\begin{aligned}
& \partial_{t} n+\frac{1}{q} \operatorname{div} J=D_{q q} \Delta n, \\
& \partial_{t} J+\frac{1}{q} \operatorname{div}\left(\frac{J \otimes J}{n}\right)+\frac{q k_{B} T_{0}}{m}\left(1+\frac{D_{p q}}{k_{B} T_{0}}\right) \nabla n-\frac{q^{2}}{m} n \nabla V-\frac{q \hbar^{2}}{6 m^{2}} n \nabla\left(\frac{\Delta \sqrt{n}}{\sqrt{n}}\right)
\end{aligned}
$$




$$
=-\frac{J}{\tau_{0}}+D_{q q} \Delta J
$$

to be coupled to the Poisson equation (1) and supplemented with initial conditions for $n$ and $J$. For the choice of the boundary conditions, see section 3.1. The second-order terms $D_{q q} \Delta n$ and $D_{q q} \Delta J$ can be interpreted as viscous terms. We stress the fact that they are formally derived from the Wigner-Fokker-Planck model; they are not an ad-hoc regularization of the quantum hydrodynamic model. The above viscous regularization is different from the viscous terms in the classical Navier-Stokes equations since it models the interactions of electrons and phonons in a semiconductor crystal. Usually, when applying a moment method to the Boltzmann equation, one would expect the continuity equation

$$
\partial_{t} n+\frac{1}{q} \operatorname{div} \widetilde{J}=0
$$

to hold, instead of (5). However, writing (5) as

$$
\partial_{t} n+\frac{1}{q} \operatorname{div}\left(J-q D_{q q} \nabla n\right)=0
$$

we can interpret $\widetilde{J}=J-q D_{q q} \nabla n$ as the (effective) current density.

The objective of this paper is to discretize the viscous quantum hydrodynamic equations (5)-(6) and (1) in one space dimension in order to understand the influence of the viscous terms on the behavior of the solutions. These are the first numerical results of the viscous quantum hydrodynamic model in the literature.

The stationary viscous quantum hydrodynamic equations have been analyzed in [26]. It has been shown that there exists a classical solution for so-called "weakly supersonic" states, assuming a smallness condition on the effective current density $\widetilde{J}$. The long-time behavior has been studied in [27].

For the inviscid quantum hydrodynamic model $\left(D_{q q}=0\right)$, more mathematical results are available. Existence results for the stationary and time-dependent equations, all under some smallness assumptions, have been obtained in [18, 28, 30, 32, 33, 43]. Concerning the numerical solution, in most works the quantum hydrodynamic model (including an equation for the temperature) has been considered as a perturbation of the hyperbolic Euler equations and discretized by using a hyperbolic scheme, e.g. an upwind finite-difference discretization [20] or a Runge-Kutta discontinuous Galerkin method [10, 11]. In [9] a solver for ordinary differential equations has been employed. However, hyperbolic schemes introduce numerical viscosity which may influence the behavior of the solution. In this paper we show that this is indeed the case.

We discretize the quantum hydrodynamic equations using a hyperbolic relaxation scheme and a central finite-difference approximation. We show numerically that the presence of (artificial or physical) viscosity changes significantly the behavior of the solutions. As the relaxation scheme introduces numerical viscosity around sharp gradients, the numerical solution is quite different from the solution computed by the central finite-difference method which avoids artifical viscosity. 
The originality of this paper consists in two facts. First, we make evident the influence of viscosity on the quantum hydrodynamic model. Second, we simulate a resonant tunneling diode and present current-voltage characteristics showing negative differential resistance and hysteresis effects for the viscous model. We notice that hysteresis in the current-voltage curve using the inviscid quantum hydrodynamic model has been first shown in [11].

The paper is organized as follows. In the next section we scale the equations. In section 3 the numerical methods are presented in detail and the schemes are tested. Section 4.1 is concerned with the numerical simulation of a ballistic diode and the numerical study of the inviscid and the semi-classical limit. In section 4.2 a resonant tunneling diode is simulated and static current-voltage characteristics are computed. Finally, we conclude the paper in section 5 .

\section{Scaling of the equations}

We scale the viscous quantum hydrodynamic equations (5)-(6) and (1) by introducing a characteristic length $L$ (for instance, the device diameter) and the characteristic time $\tau_{0}$ and define the characteristic density, voltage and current density, respectively, by

$$
C^{*}=\sup |C|, \quad V^{*}=\frac{k_{B} T_{0}}{q}, \quad J^{*}=\frac{q k_{B} T_{0} C^{*} \tau_{0}}{L m} \frac{L}{\iota},
$$

where $\iota$ is the mean-free path defined by $\iota^{2}=k_{B} T_{0} \tau_{0}^{2} / m$. After introducing the scaling

$$
x \rightarrow L x, \quad t \rightarrow \tau_{0} t, \quad n \rightarrow C^{*} n, \quad C \rightarrow C^{*} C, \quad V \rightarrow V^{*} V, \quad J \rightarrow J^{*} J,
$$

we obtain the scaled viscous quantum hydrodynamic equations

$$
\begin{aligned}
\partial_{t} n+\operatorname{div} J & =\nu \Delta n, \\
\partial_{t} J+\operatorname{div}\left(\frac{J \otimes J}{n}\right)+T \nabla n-n \nabla\left(V+V_{\text {ext }}\right)-\frac{\varepsilon^{2}}{2} n \nabla\left(\frac{\Delta \sqrt{n}}{\sqrt{n}}\right) & =-J+\nu \Delta J, \\
\lambda^{2} \Delta V & =n-C(x) .
\end{aligned}
$$

We have added to the left-hand side of (8) the term $n \nabla V_{\text {ext }}$ with the external potential $V_{\text {ext }}(x)$ which models heterogenous semiconductor materials (see section 4 for details). The scaled parameters are

$$
\varepsilon^{2}=\frac{1}{6}\left(\frac{L_{b}}{L}\right)^{2}, \quad \nu=\frac{1}{6}\left(\frac{L_{b}}{L}\right)^{2} \frac{L}{\iota} \frac{t^{*}}{\tau_{0}}, \quad T=1+\frac{1}{\sqrt{18} \pi} \frac{\Omega \hbar}{k_{B} T_{0}} \frac{L_{b}}{\iota}, \quad \lambda^{2}=\frac{\varepsilon_{s} k_{B} T_{0}}{q^{2} L^{2} C^{*}},
$$

and $L_{b}=\hbar / \sqrt{2 m k_{B} T_{0}}$ is the de Broglie length. We have assumed that the permittivity of the semiconductor is constant. Notice that the scaled effective temperature $T$ is the sum of the scaled temperature (which is one) and the correction $\Omega \hbar L_{b} / \sqrt{18} \pi k_{B} T_{0} \iota$. In the derivation of the Wigner-Fokker-Planck model it has been assumed that $\Omega \hbar / k_{B} T_{0}$ is of 


\begin{tabular}{|c|l|l|}
\hline Parameter & Physical meaning & Numerical value \\
\hline$q$ & elementary charge & $1.6 \cdot 10^{-19} \mathrm{As}$ \\
$m$ & effective electron mass & $0.063 \cdot 9.11 \cdot 10^{-31} \mathrm{~kg}$ \\
$k_{B}$ & Boltzmann constant & $1.380 \cdot 10^{-23} \mathrm{~J} / \mathrm{K}$ \\
$\hbar$ & reduced Planck constant & $1.055 \cdot 10^{-34} \mathrm{Js}$ \\
$\varepsilon_{s}$ & semiconductor permittivity & $12.9 \cdot 8.85 \cdot 10^{-12} \mathrm{As} / \mathrm{Vm}$ \\
$T_{0}$ & lattice temperature & $77 \mathrm{~K}$ \\
$\tau_{0}$ & momentum relaxation time & $0.9 \cdot 10^{-12} \mathrm{~s}$ \\
\hline
\end{tabular}

Table 1: Typical physical parameters for GaAs.

order one. Then the correction to the temperature is small if the mean free path is large compared to the de Broglie length.

The values of the parameters which we employ for the numerical simulations are displayed in Table 1. Using

$$
L=125 \mathrm{~nm}, \quad C^{*}=10^{24} \mathrm{~m}^{-3},
$$

the scaled parameters become

$$
\varepsilon^{2}=9.734 \cdot 10^{-4}, \quad \nu=9.935 \cdot 10^{-4}, \quad T=1.00585, \quad \lambda^{2}=3.032 \cdot 10^{-4} .
$$

\section{$3 \quad$ Numerical schemes}

We discretize the viscous quantum hydrodynamic equations (7)-(9) in the one-dimensional interval $\Omega=(0,1)$ using two methods: central finite differences and a relaxation scheme.

\subsection{Central finite difference scheme}

We introduce the uniform spatial mesh $x_{i}=i \triangle x, i=0, \ldots, N$, with $\triangle x=1 / N$ and denote by $n_{i}(t)$ and $\widetilde{V}_{i}(t)$ the numerical approximations of $n\left(x_{i}, t\right)$ and $V\left(x_{i}, t\right)+V_{\text {ext }}\left(x_{i}\right)$, respectively, $i=0, \ldots, N$. The current density $J$ is approximated by $J_{i-1 / 2}(t)$ at the mid points $x_{i-1 / 2}=x_{i}-\triangle x / 2, i=1, \ldots, N$. Central finite differences at $x_{i}$ for (7) and at $x_{i-1 / 2}$ for (8) yield the semi-discrete equations

$$
\begin{aligned}
\frac{\mathrm{d} n_{i}}{\mathrm{~d} t}= & -\frac{J_{i+1 / 2}-J_{i-1 / 2}}{\triangle x}+\nu \frac{n_{i+1}-2 n_{i}+n_{i-1}}{(\triangle x)^{2}}, \quad i=1, \ldots, N-1 \\
\frac{\mathrm{d} J_{i-1 / 2}}{\mathrm{~d} t}= & -\frac{1}{\triangle x}\left(\frac{J_{i}^{2}}{n_{i}}-\frac{J_{i-1}^{2}}{n_{i-1}}\right)-\frac{T}{\triangle x}\left(n_{i}-n_{i-1}\right)+\frac{n_{i-1}+n_{i}}{2 \triangle x}\left(\widetilde{V}_{i}-\widetilde{V}_{i-1}+Q_{i}-Q_{i-1}\right) \\
& -J_{i-1 / 2}+\nu \frac{J_{i+1}-2 J_{i}+J_{i-1}}{(\triangle x)^{2}}, \quad i=1, \ldots, N
\end{aligned}
$$

Here, $Q_{i}$ is the discrete quantum term

$$
Q_{i}=\frac{\varepsilon^{2}}{2(\triangle x)^{2}}\left(\sqrt{\frac{n_{i+1}}{n_{i}}}+\sqrt{\frac{n_{i-1}}{n_{i}}}-2\right),
$$


and $J_{i}=\left(J_{i-1 / 2}+J_{i+1 / 2}\right) / 2$. We impose the same boundary conditions as in [20]:

$$
\begin{gathered}
n_{0}=C(0), \quad n_{N}=C(1), \quad n_{-1}=n_{1}, \quad n_{N+1}=n_{N-1}, \\
J_{-1 / 2}=J_{1 / 2}, \quad J_{N+1 / 2}=J_{N-1 / 2} .
\end{gathered}
$$

The first two boundary conditions express that the total charge $C-n$ vanishes at the interval boundary.

The Poisson equation (9) is also discretized by central finite differences. The boundary conditions for the electric potential are $V_{0}=0$ and $V_{N}=U$, where $U$ is the applied potential. First we solve

$$
\lambda^{2} \frac{\psi_{i-1}+2 \psi_{i}-\psi_{i-1}}{(\triangle x)^{2}}=n_{i}-C\left(x_{i}\right), \quad i=1, \ldots, N-1
$$

with $\psi_{0}=0$ and $\psi_{1}=0$. The potential $V_{i}$ satisfying the boundary condition at $x_{N}$ is then given by $V_{i}=\psi_{i}+i\left(U-\psi_{N}\right) / N$, and the potential $\widetilde{V}_{i}=V_{i}+V_{\text {ext }}\left(x_{i}\right)$.

We solve (11)-(12) with an explicit second-order Runge-Kutta method. For each time step, after solving (11)-(12), the Poisson equation (14) is solved using the new value for $n_{i}$. The stationary solution is computed as the long-time limit of the transient solution. The time-step control is done heuristically, and the transient computations are stopped when the changes of the average effective current and of the effective current density at selected points are smaller than a certain tolerance. We recall that the effective current density $J-\nu \partial_{x} n$ is constant in the stationary viscous model. At applied voltage $U=0$, the initial conditions for (11)-(12) may be chosen as $n(x, 0)=C(x)$ and $J(x, 0)=0$. After obtaining the solution at applied voltage $U$ we use this solution as initial data for (11)-(12) with an applied voltage $U+\triangle U$ for some increment $\triangle U$ (continuation method).

The described scheme cannot be used for the inviscid problem $(\nu=0)$ since there is essentially no viscosity in the central finite difference discretization, whereas the physical viscosity $\nu$ stabilizes the central scheme. In order to compute also solutions of the inviscid stationary problem we use the same discretization as above but we solve the nonlinear equations by employing the standard Newton-Raphson method.

\subsection{Relaxation scheme}

The system (7)-(8) can be seen as hyperbolic conservation laws (including potential terms as well as dissipation and diffusion). The eigenvalues of the hyperbolic part are

$$
\lambda_{ \pm}=\frac{J}{n} \pm \sqrt{T}
$$

In order to solve this hyperbolic system, we adapt a relaxation scheme based on the pioneering work of Jin and Xin [29] and further developed, e.g., in [2]. The relaxation scheme proves to be an appropriate method for solving the classical hydrodynamic semiconductor equations [35]. For these equations, also other numerical schemes have been employed, 
e.g., second-order upwind shock-capturing methods [19], the Runge-Kutta discontinuous Galerkin method [12], or ENO (essentially non-oscillatory) schemes [40].

More precisely, we write the equations (7)-(8) as a hyperbolic system with source terms

$$
\partial_{t} U+\partial_{x} A(U)=\left(f^{1}, f^{2}\right)^{\top}
$$

where $U=(n, J), A(U)=\left(J, J^{2} / n+T n\right)^{\top}$,

$$
f^{1}=\nu \partial_{x x} n, \quad f^{2}=-J+n \partial_{x}(\widetilde{V}+Q)+\nu \partial_{x x} J
$$

and $Q=\varepsilon^{2}\left(\partial_{x x} \sqrt{n}\right) / 2 \sqrt{n}$. We discretize the convective system

$$
\partial_{t} U+\partial_{x} A(U)=0
$$

in a uniform spatial grid as in the previous subsection with grid points $x_{i}=i \triangle x$ and grid size $\triangle x>0$ and assign a linear propagation speed matrix $\Lambda=\operatorname{diag}\left(\lambda_{1}, \lambda_{2}\right)$ with suitable constants $\lambda_{1}, \lambda_{2}>0$ satisfying the subcharacteristic condition (see [2]). Denoting by $U_{i}$ an approximation of $U\left(x_{i}\right)$, a semi-discrete relaxed scheme with minmod limiter reads as follows:

$$
\frac{\mathrm{d} U_{i}}{\mathrm{~d} t}=\frac{A\left(U_{i-1}\right)-A\left(U_{i+1}\right)}{2 \triangle x}-\frac{1}{2 \triangle x} \Lambda B_{i},
$$

where

$$
\begin{aligned}
B_{i}= & \operatorname{minmod}\left(M_{i+1}^{+}-M_{i}^{+}, M_{i}^{+}-M_{i-1}^{+}\right)-\operatorname{minmod}\left(M_{i}^{+}-M_{i-1}^{+}, M_{i-1}^{+}-M_{i-2}^{+}\right) \\
& +\operatorname{minmod}\left(M_{i+2}^{-}-M_{i+1}^{-}, M_{i+1}^{-}-M_{i}^{-}\right)-\operatorname{minmod}\left(M_{i+1}^{-}-M_{i}^{-}, M_{i}^{-}-M_{i-1}^{-}\right),
\end{aligned}
$$

and

$$
M_{i}^{ \pm}=\frac{1}{2}\left(U_{i} \pm \Lambda^{-1} A\left(U_{i}\right)\right) .
$$

Then we take into account the source terms $f^{1}$ and $f^{2}$ which are discretized with central finite differences; i.e., with $f_{i}^{1}$ and $f_{i}^{2}$ denoting the approximations of $f^{1}$ and $f^{2}$ at $x_{i}$, respectively, we approximate

$$
\begin{aligned}
f_{i}^{1} & =\nu \frac{n_{i+1}-2 n_{i}+n_{i-1}}{(\triangle x)^{2}} \\
f_{i}^{2} & =-J_{i}+\frac{n_{i}}{2 \triangle x}\left(\widetilde{V}_{i+1}-\widetilde{V}_{i-1}+Q_{i+1}-Q_{i-1}\right)+\nu \frac{J_{i+1}-2 J_{i}+J_{i-1}}{(\triangle x)^{2}}
\end{aligned}
$$

and $Q_{i}$ is given by (13). We end up with the ODE system

$$
\frac{\mathrm{d} U_{i}}{\mathrm{~d} t}=\frac{A\left(U_{i-1}\right)-A\left(U_{i+1}\right)}{2 \triangle x}-\frac{1}{2 \triangle x} \Lambda B_{i}+\left(f_{i}^{1}, f_{i}^{2}\right)^{\top}
$$

which is solved by an explicit second-order Runge-Kutta method. We use the same set of boundary conditions as in the previous subsection. 


\subsection{Numerical tests}

In this section we perform a numerical test for the two schemes proposed in the previous subsections. We use as test problem a GaAs ballistic $n^{+} n n^{+}$device with length $L=125 \mathrm{~nm}$ and without external potential $\left(V_{\text {ext }}=0\right)$. The channel length is $25 \mathrm{~nm}$ and the doping profile $C(x)$ is slightly smoothed around the junctions at $x=50 \mathrm{~nm}$ and $x=75 \mathrm{~nm}$ :

$$
C(x)=[1+0.4995(\tanh (10 x-750)-\tanh (10 x-500))] \cdot 10^{24} \mathrm{~m}^{-3}, \quad x \in[0,125 \mathrm{~nm}] .
$$

The scaled parameters are given by (10).
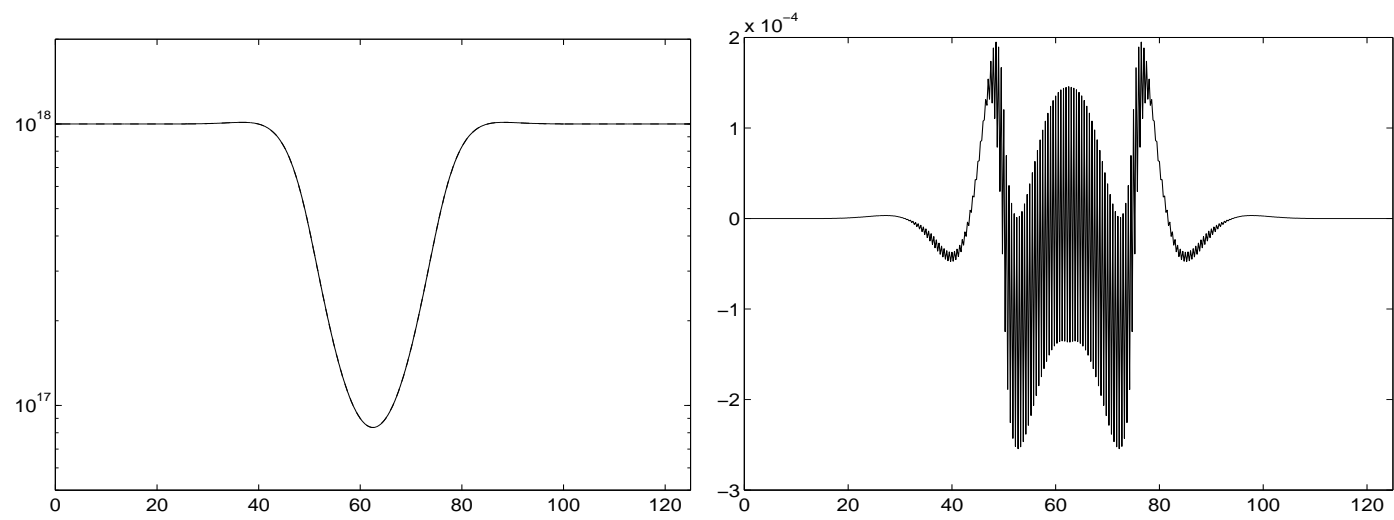

Figure 1: Electron density (in $\mathrm{cm}^{-3}$ ) versus position (in $\mathrm{nm}$ ), computed by the relaxation scheme (left) and relative error between the relaxation scheme and the Newton-Raphson scheme (right) using 500 grid points.

First we solve the inviscid problem $(\nu=0)$ in thermal equilibrium $(U=0)$ using the relaxation scheme. We choose the (unscaled) relaxation parameters $\lambda_{1}=0.05$ and $\lambda_{2}=80$ (in units of $10^{5} \mathrm{~m} / \mathrm{s}$ ). In Figure 1 (left) the stationary electron density (obtained as the long-time "limit" of the transient solution) is shown using 500 points. For comparison we have also computed the particle density employing a standard Newton-Raphson scheme as explained in section 3.1. In Figure 1 (right) the relative error of both solutions is shown. The relative error is smaller than $3 \cdot 10^{-4}$ with a grid size of $\triangle x=2 \cdot 10^{-3}$. The relative error between the relaxation scheme and the central finite-difference scheme (stabilized with a very small viscosity) is of the same order. We remark that the oscillatory behavior of the relative error comes from the dispersive leading-order "error" in the modified equation for the numerical scheme.

It is worth mentioning that the choice of the relaxation parameters $\lambda_{1}$ and $\lambda_{2}$ can be delicate. While they should be chosen large enough to maintain stability, the numerical viscosity increases with increasing parameters [2]. For instance, fixing $\lambda_{2}=80$, we compute the particle and current densities for various values of $\lambda_{1}$. Whereas the particle densities are very similar (as in Figure 1), the current density changes significantly with different $\lambda_{1}$ (Figure 2). For larger $\lambda_{1}$ we observe larger values of the current density, caused by the numerical viscosity. On the other hand, for smaller $\lambda_{1}$ we observe an increasing oscillation 
frequency. A reasonable choice of $\lambda_{1}$ and $\lambda_{2}$ requires a balance between dispersion and dissipation for which, up to now, no theoretical result is available as a guide. On the other hand, the effective current density $\widetilde{J}$ computed by the Newton scheme is a constant and, up to rounding errors, equal to zero.

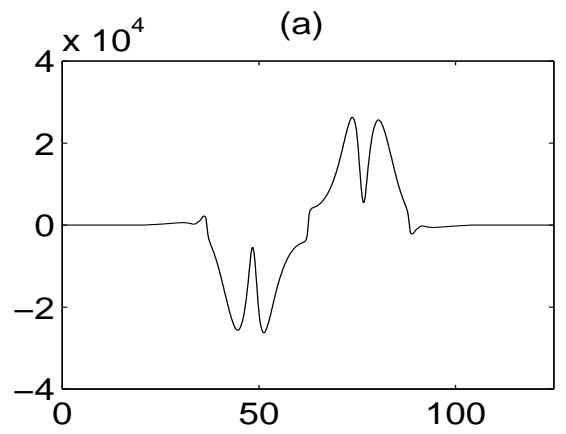

(c)

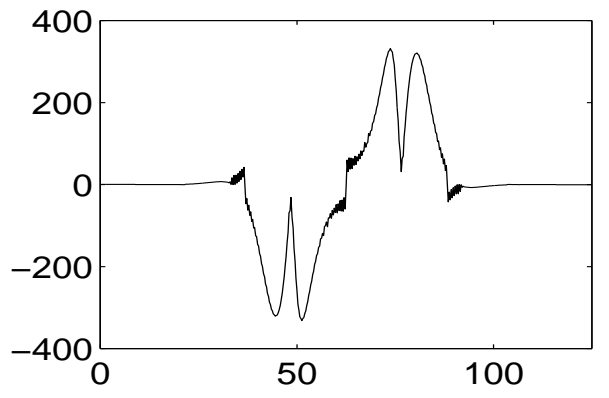

(b)

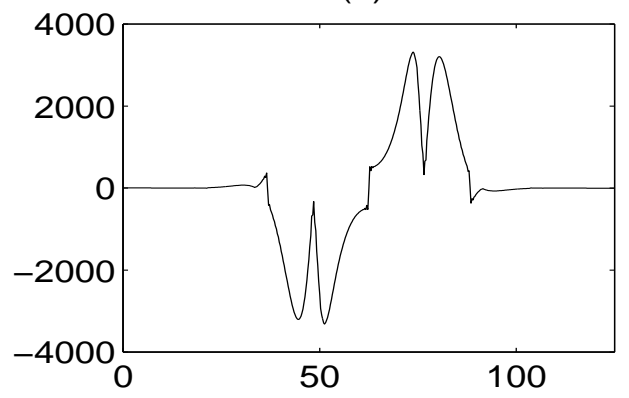

(d)

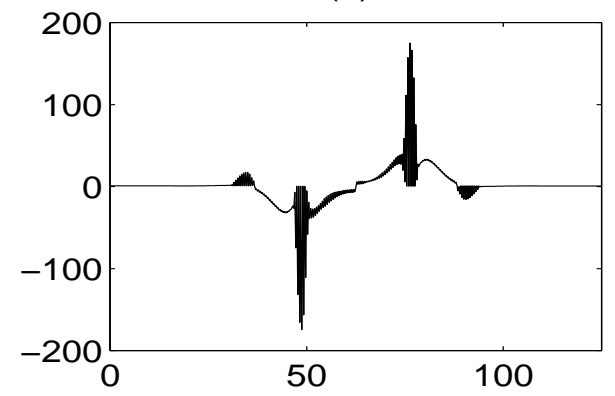

Figure 2: Current density (in $\mathrm{A} / \mathrm{cm}^{2}$ ) versus position (in $\mathrm{nm}$ ), computed by the relaxation scheme with the relaxation parameter $\lambda_{2}=80$ and various values of $\lambda_{1}$ (in units of $10^{5} \mathrm{~m} / \mathrm{s}$ ): (a) $\lambda_{1}=80$, (b) $\lambda_{1}=10$, (c) $\lambda_{1}=1$, (d) $\lambda_{1}=0.05$. In (c) and (d), unphysical highfrequency oscillations can be observed.

The above results show that the numerical solution of the equations using the relaxation scheme may be delicate. This observation becomes more important for positive applied voltage. For instance, even at the rather low applied voltage $U=0.05 \mathrm{~V}$, the particle density is quite different for various values of the relaxation parameters $\lambda_{1}$ and $\lambda_{2}$ (Figure 3 ). For smaller relaxation parameters, more oscillations are observed. This also holds true for the current density, depicted in Figure 4. Moreover, the (average) value of the current density depends on the relaxation parameters.

We summarize that the behavior of the solution of the inviscid model depends strongly on the artificial viscosity of the relaxation scheme. Clearly, it also influences the solution of the viscous model, depending on the order of magnitudes of the numerical and the physical viscosity. Therefore, in the following simulations we have only used the central finite-difference scheme. 
(a)

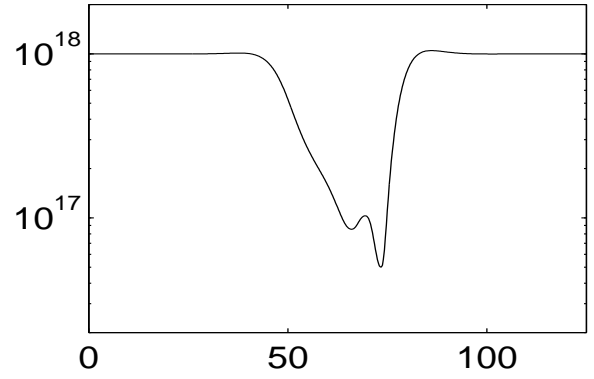

(c)

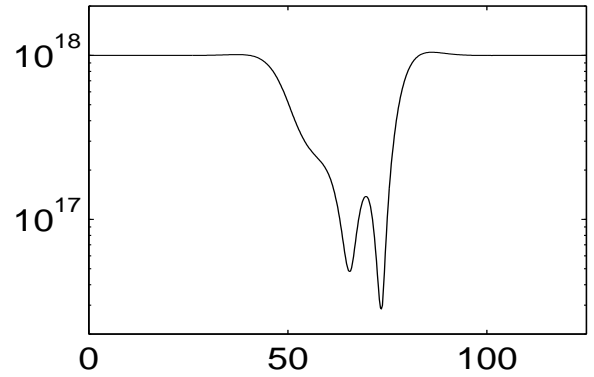

(b)

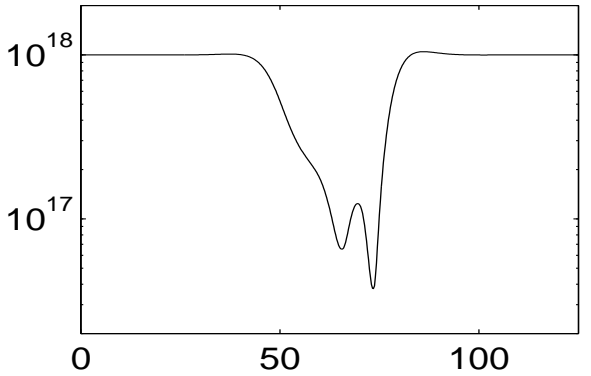

(d)

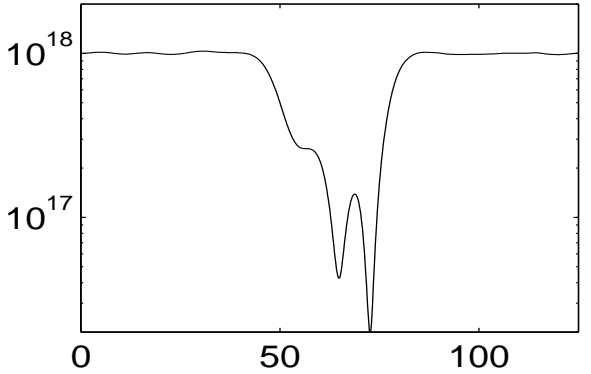

Figure 3: Electron density (in $\mathrm{cm}^{-3}$ ) versus position (in $\mathrm{nm}$ ), computed by the relaxation scheme: (a) $\left(\lambda_{1}, \lambda_{2}\right)=(80,80)$; (b) $\left(\lambda_{1}, \lambda_{2}\right)=(40,80)$; (c) $\left(\lambda_{1}, \lambda_{2}\right)=(20,40)$; (d) $\left(\lambda_{1}, \lambda_{2}\right)=$ $(10,20)$. The values of $\lambda_{1}$ and $\lambda_{2}$ are in units of $10^{5} \mathrm{~m} / \mathrm{s}$.

\section{Numerical simulations}

\subsection{Numerical simulation of a ballistic diode}

In order to understand the influence of the quantum term as well as the (physical) viscosity we simulate a ballistic $n^{+} n n^{+}$diode. We wish to compare our results to those of [38]. Therefore, we replace the term $-J$ on the right-hand side of (8) by $-J / \tau$ and choose the scaled parameters

$$
\varepsilon=0.00289, \quad \lambda=0.1, \quad \tau=0.125
$$

and the doping profile

$$
C(x)=1+0.45(\tanh (1000 x-600)-\tanh (1000 x-400)), \quad x \in(0,1) .
$$

First we study the inviscid quantum hydrodynamic equations. It is well known that the classical hydrodynamic model $(\varepsilon=0)$ can develop a shock discontinuity in the transition from a supersonic region to a subsonic region, while the transition from the subsonic to the supersonic region is smooth [5]. As the sound speed in the scaled system is one, $J<n$ characterizes the subsonic region and $J>n$ the supersonic zone. The two regions are separated by the sonic line $n=J$ (notice that $J$ is constant in the stationary inviscid problem). 

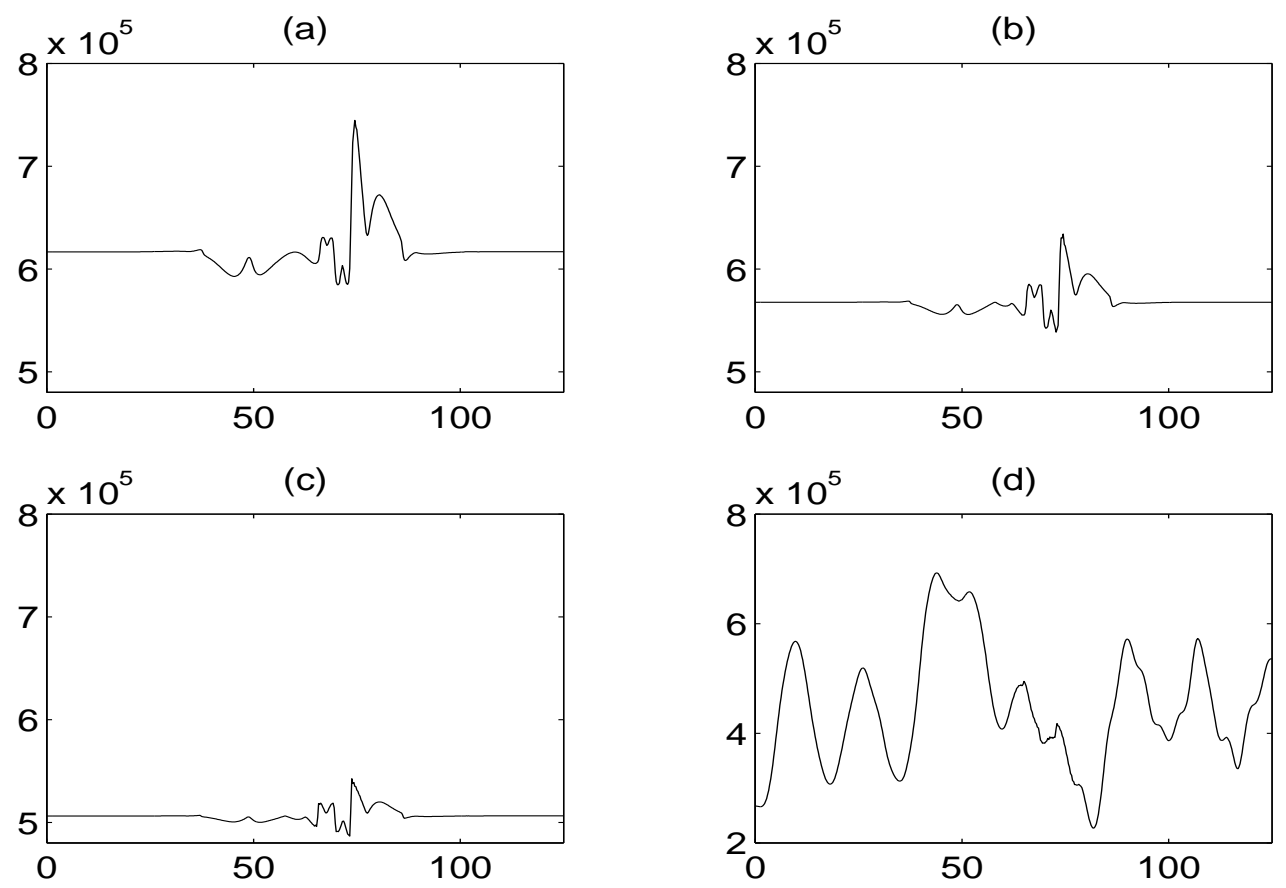

Figure 4: Current density (in $\mathrm{A} / \mathrm{cm}^{2}$ ) versus position (in $\mathrm{nm}$ ), computed by the relaxation scheme: (a) $\left(\lambda_{1}, \lambda_{2}\right)=(80,80)$; (b) $\left(\lambda_{1}, \lambda_{2}\right)=(40,80)$; (c) $\left(\lambda_{1}, \lambda_{2}\right)=(20,40)$; (d) $\left(\lambda_{1}, \lambda_{2}\right)=$ $(10,20)$. The values of $\lambda_{1}$ and $\lambda_{2}$ are in units of $10^{5} \mathrm{~m} / \mathrm{s}$.

In Figure 5 we present the electron density for different values of the applied voltage. In the subsonic zone $n>J$ the solution is smooth whereas in the supersonic zone $n<J$, the particle density oscillates with a wave length of order $\varepsilon$ [38]. The oscillations are not a numerical artefact as already pointed out in [38]. In fact, they are due to the dispersive quantum term and double grid simulations give the same result. Comparing the above results with the simulations using the viscous model with scaled viscosity $\nu=4.267 \cdot 10^{-3}$ (Figure 6), we observe that the oscillatory pattern is dissipated. The wave length is the same as for the inviscid model, but the amplitude is smaller.

In the following we are interested in two asymptotic limits: the inviscid limit $\nu \rightarrow 0$ and the semi-classical limit $\varepsilon \rightarrow 0$. In [26] it has been proved that the solutions converge for $\nu \rightarrow 0, \varepsilon \rightarrow 0$, respectively, to a solution of the limit problem (the inviscid quantum hydrodynamic model or the viscous hydrodynamic model, respectively) under the assumption that the current density is small enough. We want to present numerical results for large current density or large applied voltage.

In Figure 7 the particle densities for various viscosities are displayed. The (scaled) applied voltage is chosen to be $U=5$, and the solutions are computed using the central scheme of section 3.1. We observe that the oscillations are smeared out for larger viscosity and vanish completely for large enough viscosities. This is more pronounced close to the left junction $x=0.4$ where the particles traveling from left to right enter the supersonic region. For $\nu=0.125 \nu_{0} \approx 5 \cdot 10^{-5}$ the solution almost coincides with the solution of the 
(a)

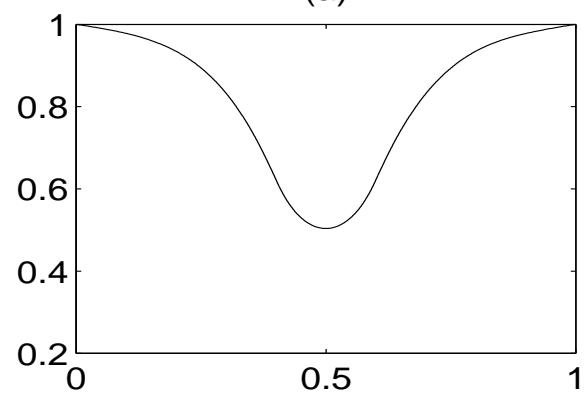

(c)

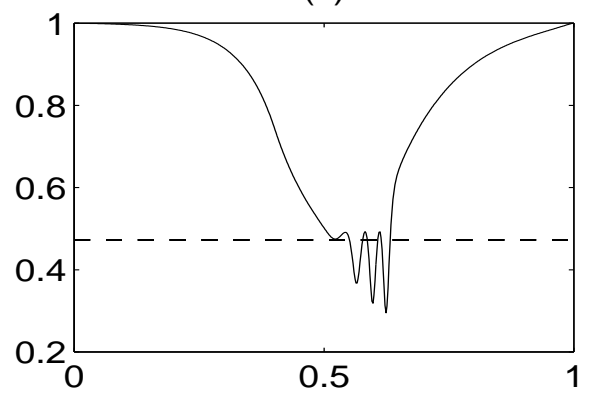

(b)

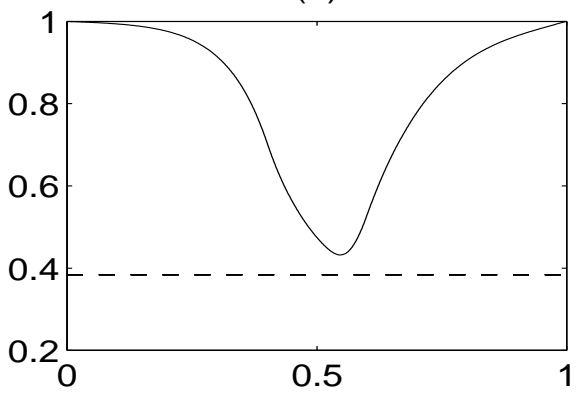

(d)

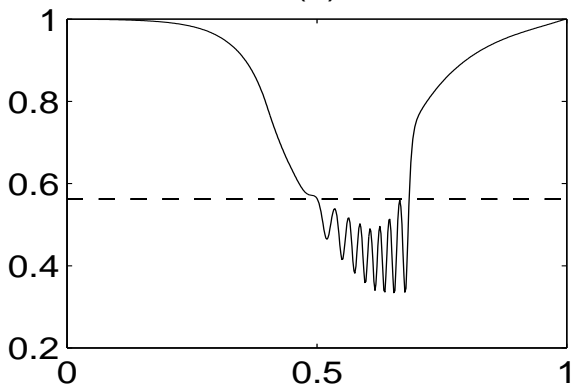

Figure 5: Electron density versus position of the inviscid quantum hydrodynamic model, computed by the Newton method. The dashed line indicates the value of the current density and separates the subsonic and supersonic regions. The (scaled) applied voltages are (a) $U=0$; (b) $U=4$; (c) $U=5$; (d) $U=6$.

inviscid model in Figure 5.

The current density $J$ also converges to the current density of the inviscid model as the viscosity tends to zero (Figure 8). Notice that the variable $J$ is not constant in the viscous model. However, the effective current density $J-\nu \partial_{x} n$ is a constant. Therefore, we expect the variable $J$ to converge to the constant current density of the inviscid model. In Figure 8 the current density of the inviscid model is computed using the Newton method.

In the semi-classical limit $\varepsilon \rightarrow 0$, the electron density seems to converge to a solution with a very sharp gradient near $x=0.6$ (Figure 9 ). This is in contrast to the convergence behavior of the solution to the inviscid model. Indeed, for smaller values of $\varepsilon$, the frequency of the oscillations increases, and it has been shown numerically in [38] that the solution of the inviscid quantum hydrodynamic equations do not converge weakly to the solution of the hydrodynamic model (in the transonic case). Therefore, the viscous terms seem to regularize the equations in the semi-classical limit.

Another behavior is expected if both $\varepsilon$ and $\nu$ tend to zero. In this situation the limit are the classical hydrodynamic equations. We let $\nu$ tend to zero faster than $\varepsilon$ by changing $\nu$ and $\varepsilon$ by $\nu \cdot \gamma^{2}$ and $\varepsilon \cdot \gamma$, respectively, and let $\gamma \rightarrow 0$. Figure 10 for $U=5$ shows that the frequency of the oscillations of the electron density increases in the limit, and no (strong) limit can be expected, similar as for the semi-classical limit in the inviscid model. Again, the oscillations are not a numerical artefact as a double grid computation shows a similar 
(a)

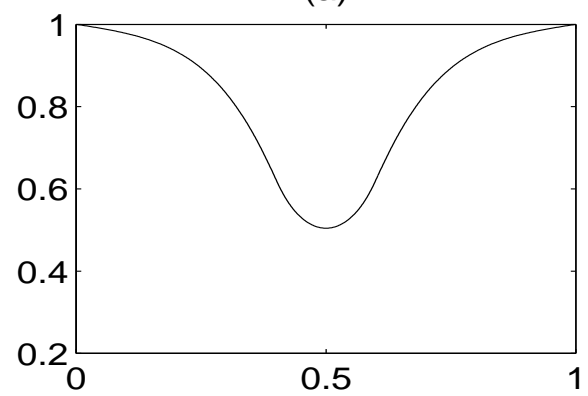

(c)

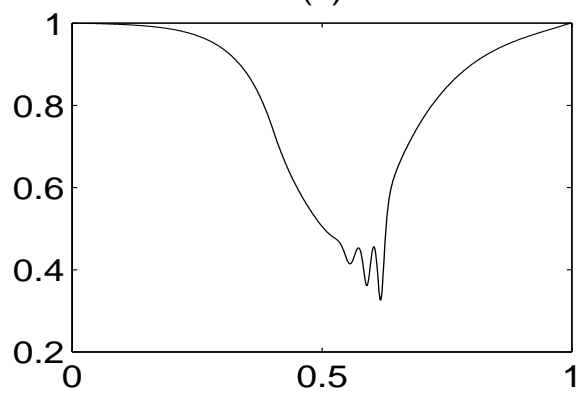

(b)

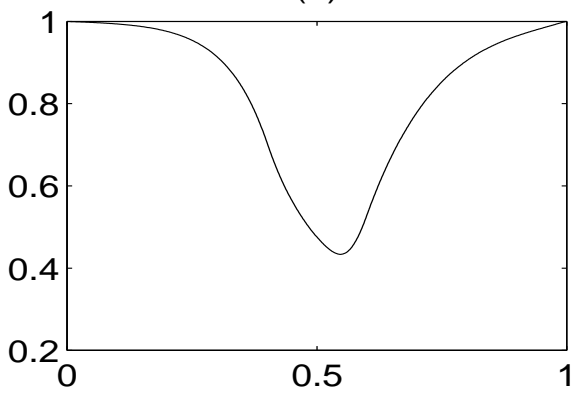

(d)

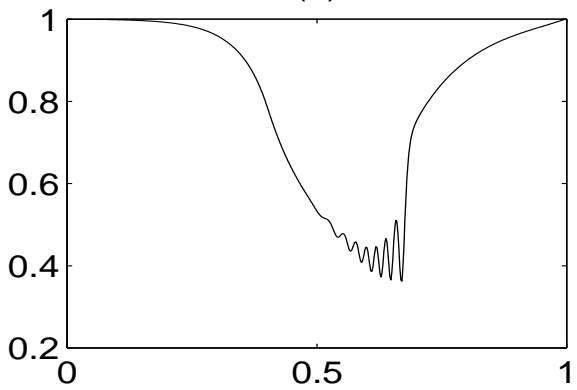

Figure 6: Electron density versus position of the viscous quantum hydrodynamic model, computed by the central finite-difference scheme of section 3.1. The scaled applied voltages are (a) $U=0$; (b) $U=4$; (c) $U=5$; (d) $U=6$.

behavior. Due to the high-frequency oscillations we need a refined mesh for subplots (c) and (d). For instance, we use 4000 grid points in subplot (d).

In [26] it has been shown that the combined limit $\varepsilon \rightarrow 0$ and $\nu \rightarrow 0$ can be performed rigorously in the "subsonic" viscous model. In particular, the solutions converge strongly in some Lebesgue space. Figure 10 shows that strong convergence of the particle densities seems not to hold in the "supersonic" case.

\subsection{Simulation of a resonant tunneling diode}

In this section we simulate a one-dimensional resonant tunneling diode using the viscous quantum hydrodynamic model. The tunneling diode consists of highly doped GaAs regions near the contacts and a lightly doped middle region of $50 \mathrm{~nm}$ length. The middle region contains a quantum well of $5 \mathrm{~nm}$ length sandwiched between two $5 \mathrm{~nm}$ AlGaAs barriers. This resonant barrier structure is itself sandwiched between two $5 \mathrm{~nm}$ GaAs spacer layers. The physical effect of the barriers is a shift in the quasi-Fermi level which can be modeled by an additional step function $V_{\text {ext }}$ added to the electrostatic potential. More precisely, the device length is $L=125 \mathrm{~nm}$, the doping profile has a sharp discontinuity,

$$
C(x)=\left\{\begin{aligned}
5 \cdot 10^{21} \mathrm{~m}^{-3} & \text { for } 50 \mathrm{~nm} \leq x \leq 75 \mathrm{~nm} \\
10^{24} \mathrm{~m}^{-3} & \text { else }
\end{aligned}\right.
$$


(a)

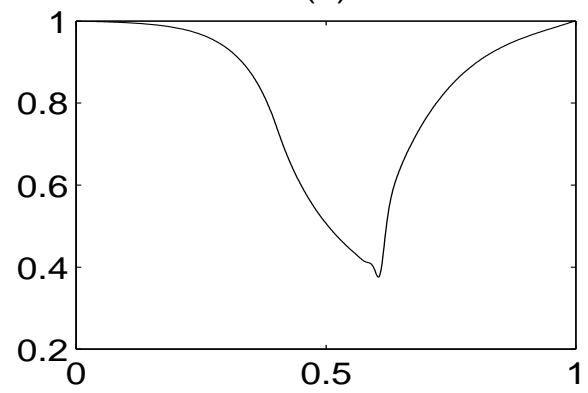

(c)

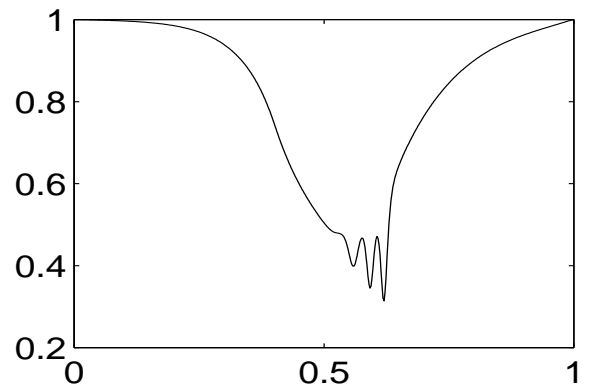

(b)

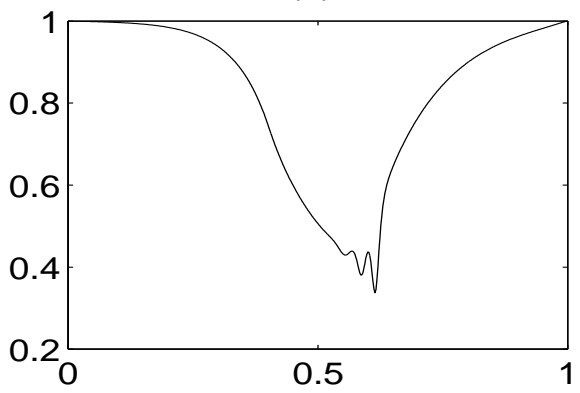

(d)

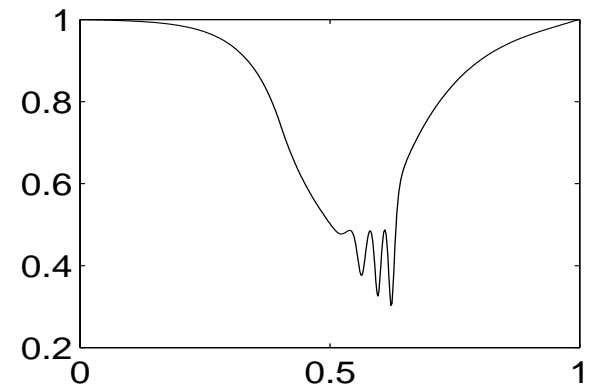

Figure 7: Inviscid limit in the viscous quantum hydrodynamic model. Electron density versus position for applied voltage $U=5$ and viscosities (a) $\nu=8 \nu_{0}$; (b) $\nu=2 \nu_{0}$; (c) $\nu=0.5 \nu_{0} ;$ (d) $\nu=0.125 \nu_{0}$ with $\nu_{0}=4.267 \cdot 10^{-3}$.

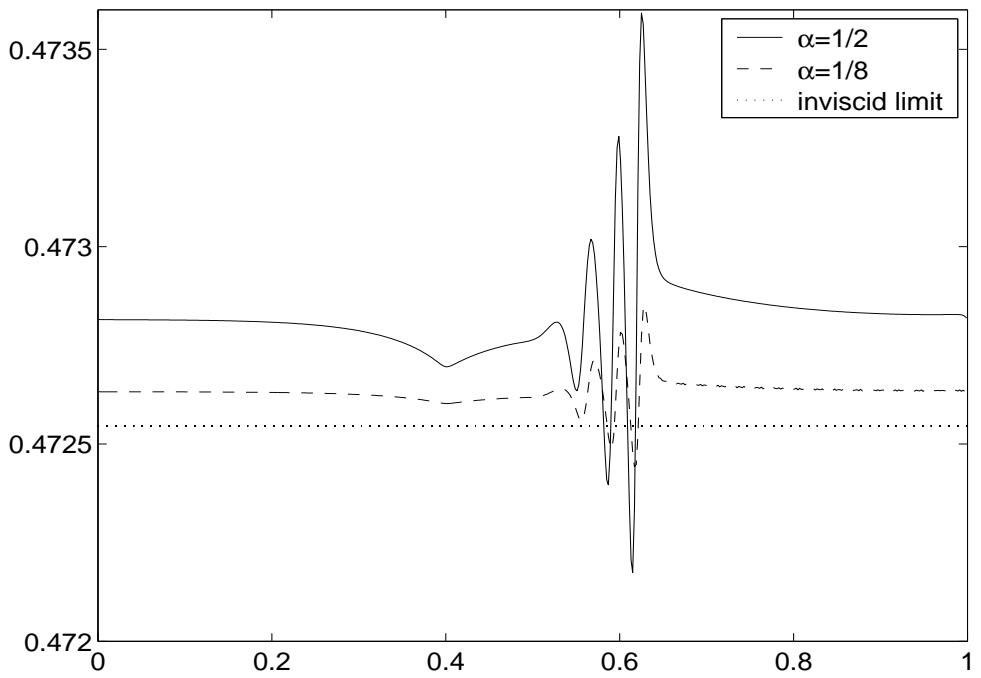

Figure 8: Inviscid limit in the viscous quantum hydrodynamic model. Current density versus position for applied voltage $U=4$ and viscosities $\nu=\alpha \nu_{0}$ with $\nu_{0}=4.267 \cdot 10^{-3}$. Notice that the variable $J$ is not constant in the viscous model. 
(a)

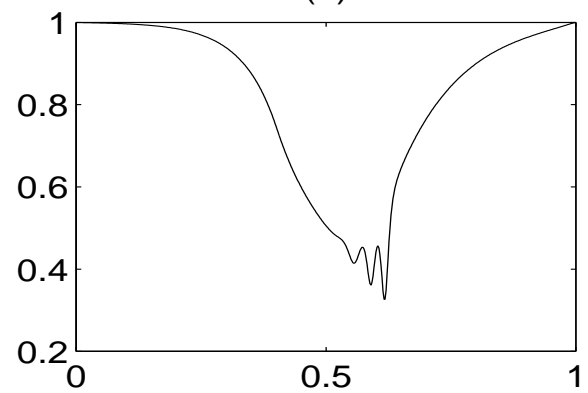

(c)

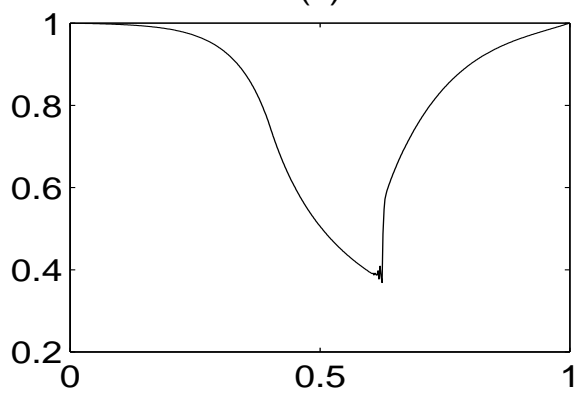

(b)

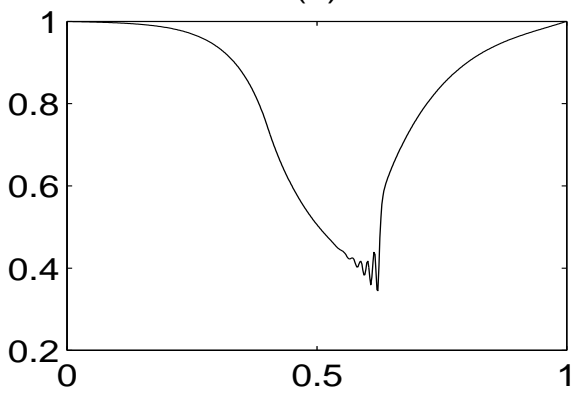

(d)

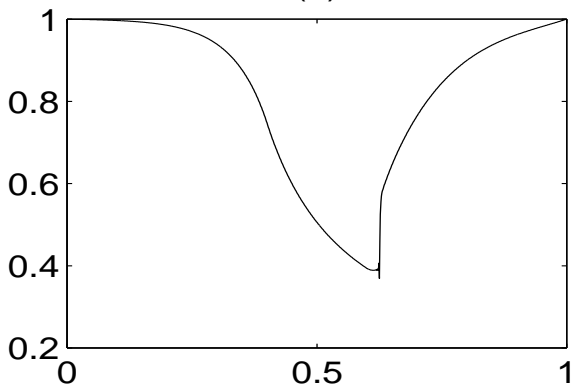

Figure 9: Semi-classical limit in the viscous quantum hydrodynamic model. Electron density versus position with $\nu=4.267 \cdot 10^{-3}, U=5$ and (a) $\varepsilon=\varepsilon_{0}$; (b) $\varepsilon=0.5 \varepsilon_{0}$; (c) $\varepsilon=0.25 \varepsilon_{0} ;(\mathrm{d}) \varepsilon=0.125 \varepsilon_{0}$, where $\varepsilon_{0}=0.00289$.

and the external potential $V_{\text {ext }}$ is taken as in [20]:

$$
V_{\text {ext }}(x)=\left\{\begin{aligned}
-0.209 \mathrm{~V} & \text { for } x \in[55 \mathrm{~nm}, 60 \mathrm{~nm}] \cup[65 \mathrm{~nm}, 70 \mathrm{~nm}] \\
0 & \text { else. }
\end{aligned}\right.
$$

We are interested in the stationary current-voltage characteristics. For the numerical computations we employ the continuation method. More precisely, we choose the stationary solution of the hydrodynamic model (see [35]) as the initial data for zero applied voltage. The solution converges numerically to a stationary solution of the viscous quantum model. After obtaining a stationary solution at applied voltage $U$, we use it as the initial data for the computation with applied voltage $U+\triangle U$, with a small non-uniform increment $\triangle U$. We take in the simulations 500 grid points and a time step of $10^{-5} \mathrm{ps}$. The transient computations are stopped when the changes of the average current and of the current density at selected points are smaller than $0.1 \%$. The time to reach the steady state depends on the increment $\triangle U$, but typically, the equilibrium is reached in 10 to $20 \mathrm{ps}$. We recall that the relaxation time equals $\tau_{0}=0.9 \mathrm{ps}$.

The numerical current-voltage characteristic in Figure 11 is computed by first increasing the applied voltage from $U=0 \mathrm{~V}$ to $U=0.3 \mathrm{~V}$ and then, choosing the solution for the largest applied voltage as the initial data, by decreasing the applied voltage. More precisely, the curve displays the effective current density $J-\nu \partial_{x} n$ versus the applied voltage since the variable $J$ is not constant in the viscous model. 
(a)

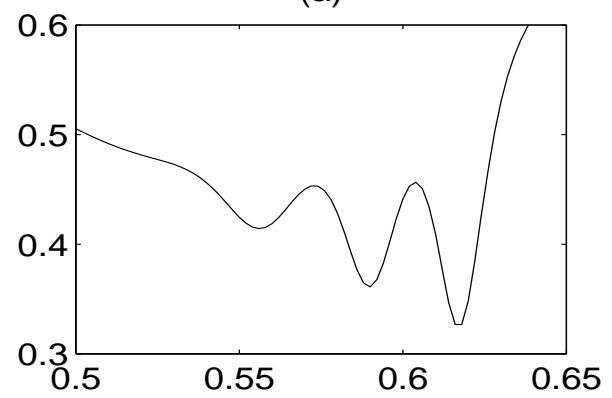

(c)

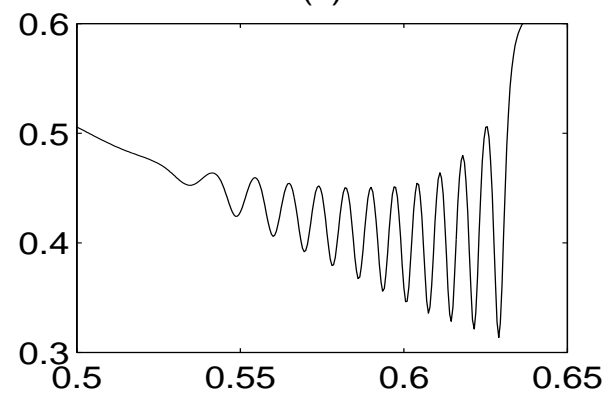

(b)

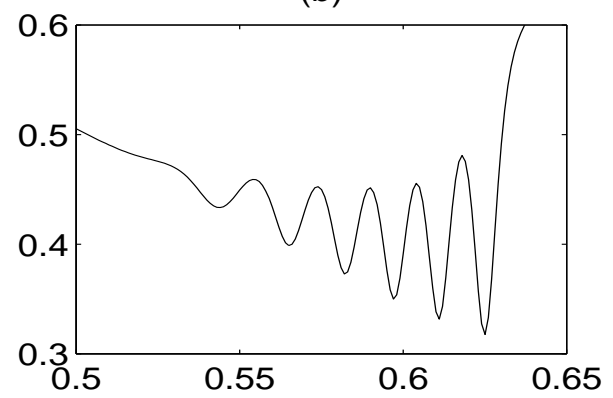

(d)

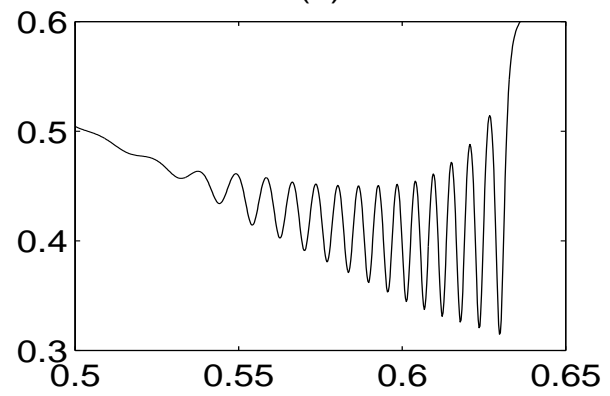

Figure 10: Combined inviscid and semi-classical limit in the viscous model. Electron density versus position for $U=5, \nu=\nu_{0} \gamma^{2}$ and $\varepsilon=\varepsilon_{0} \gamma$, where $\nu_{0}=4.267 \cdot 10^{-3}$ and $\varepsilon_{0}=0.00289$. (a) $\gamma=1$; (b) $\gamma=1 / 2$; (c) $\gamma=1 / 4$; (d) $\gamma=1 / 5$.

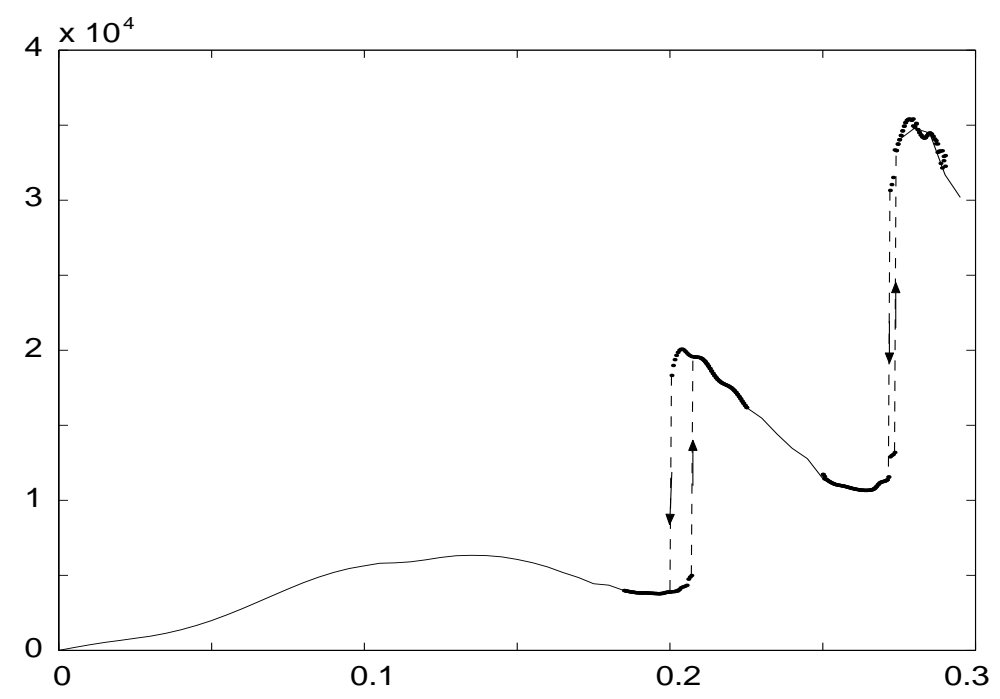

Figure 11: Effective current density (in $\mathrm{A} / \mathrm{cm}^{-2}$ ) versus applied voltage (in $\mathrm{V}$ ) for a resonant tunneling diode. The arrows indicate the direction of increasing or decreasing applied voltage. 
The characteristic shows several features. First, there are regions in which the effective current density is decreasing with increasing applied voltage. These regions have a negative differential resistance and characterize the tunneling diode. Multiple regions of negative differential resistance in the inviscid model have been first observed in [21]. Second, the curve has very sharp gradients just before the regions of negative differential resistance. More precisely, the jumps occur when the applied voltage is increased from $U=0.2070 \mathrm{~V}$ to $U=0.2075 \mathrm{~V}$ and from $U=0.2735 \mathrm{~V}$ to $U=0.2740 \mathrm{~V}$. Physically, we expect sharp gradients just after the local maximum of the current density and not before. Why the viscous quantum hydrodynamic model shows a different behavior is under investigation. A possible reason could be that the temperature is assumed to be constant. In fact, the usual quantum hydrodynamic simulations in the literature (e.g., [11, 20, 23]) always include the energy equation.

In Figure 12 the particle densities just before and after the jump are shown. We observe that the electron density develops a "wiggle" when the current density jumps. For decreasing applied voltage, the jumps occur at smaller values of $U$. In fact, the curve shows a hysteresis effect. Hysteresis in the current-voltage curve of the inviscid quantum hydrodynamic model has been already observed in [11]. The reported characteristic is different from our results since the authors in [11] do not observe jumps in the curve. However, in [11] a different model is used (the temperature equation is included) and a hyperbolic solver is used which introduces non-uniform numerical viscosity. Therefore, it is not surprising that hysteresis also occurs in our viscous model. Mathematically, hysteresis indicates non-uniqueness of solutions. There are values of the applied voltage for which there are at least two solutions (Figure 13).
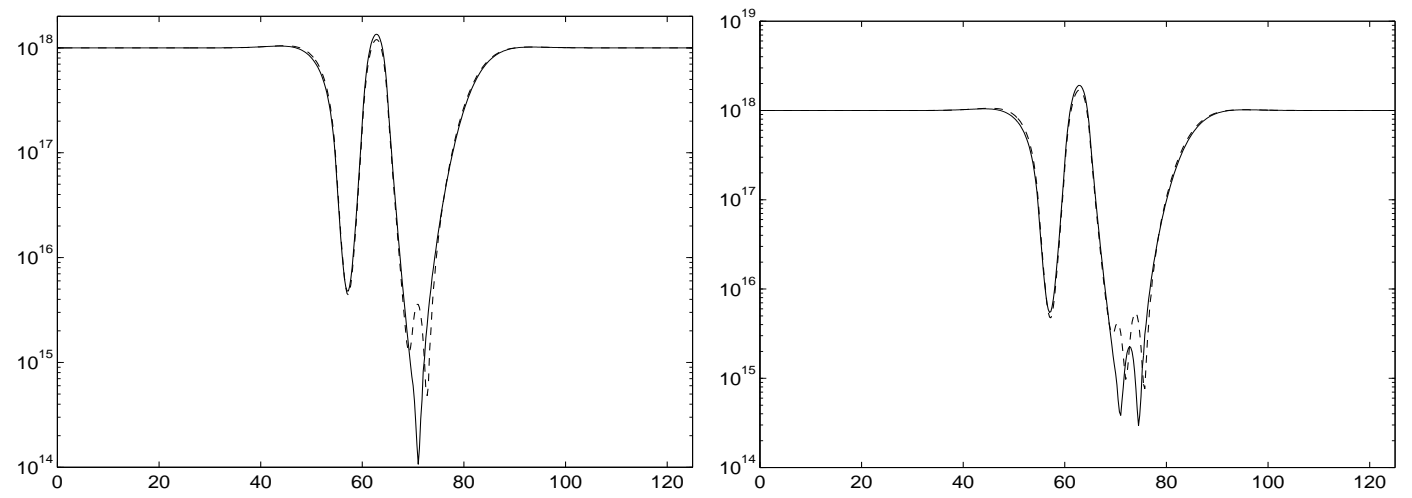

Figure 12: Electron density (in $\mathrm{cm}^{-3}$ ) versus position (in $\mathrm{nm}$ ) in a resonant tunneling diode. Left figure: $U=0.2070 \mathrm{~V}$ (solid line) and $U=0.2075 \mathrm{~V}$ (dashed line). Right figure: $U=0.2735 \mathrm{~V}$ (solid line) and $U=0.2740 \mathrm{~V}$ (dashed line).

The influence of the viscosity on the current-voltage characteristics is shown in Figure 14. We clearly see that the sharp gradients are smoothed by the viscous effects. Although the viscosity constant is only changed by a factor two, the curves are quite different which shows that the models depend very sensitively on the viscosity constant. This observation 


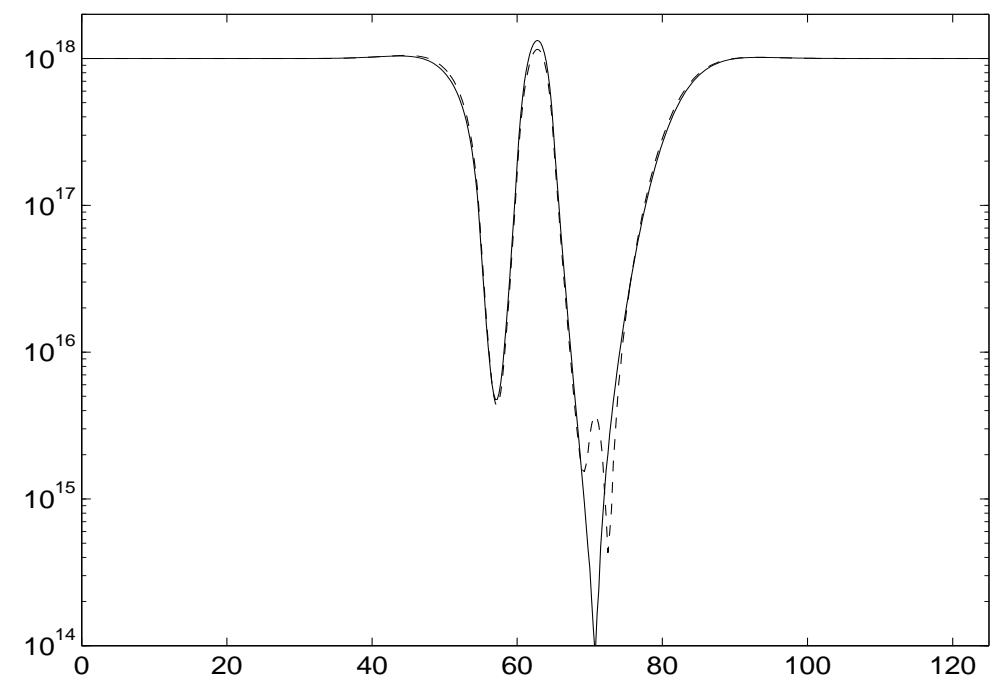

Figure 13: Electron densities (in $\mathrm{cm}^{-3}$ ) versus position (in $\mathrm{nm}$ ) in a resonant tunneling diode at $U=0.204 \mathrm{~V}$ (solid line for increasing applied voltage and dashed line for decreasing applied voltage).

indicates that the use of hyperbolic solvers with non-uniform artificial viscosity, applied to the quantum hydrodynamic equations, has to be done with care.

The final Figure 15 is concerned with the influence of the lattice temperature. A larger temperature constant seems also to smoothen the current-voltage curve. We expect that for larger lattice temperature, the thermal diffusion of the electrons destroys the resonant effects, and no regions with negative differential resistance can be observed anymore.

\section{Conclusions}

We have discretized the inviscid and the viscous quantum hydrodynamic equations using a central finite-difference and a relaxation scheme. Numerical tests showed that the relaxation scheme seems to be inappropriate to solve the equations numerically since the numerical viscosity changes the behavior of the solutions significantly. On the other hand, the central scheme avoids any artifical viscosity.

The inviscid limit and the semi-classical limit have been performed numerically in the viscous quantum model. It turns out that the solution converges numerically to the solution of the limit model even in the supersonic zones. However, in the combined limit, the particle density develops high-frequency oscillations and no (strong) convergence can be expected.

Finally, we simulated a resonant tunneling structure. The current-voltage characteristic shows negative differential resistance and hysteresis effects. Moreover, the viscosity terms make the characteristics "smoother". 


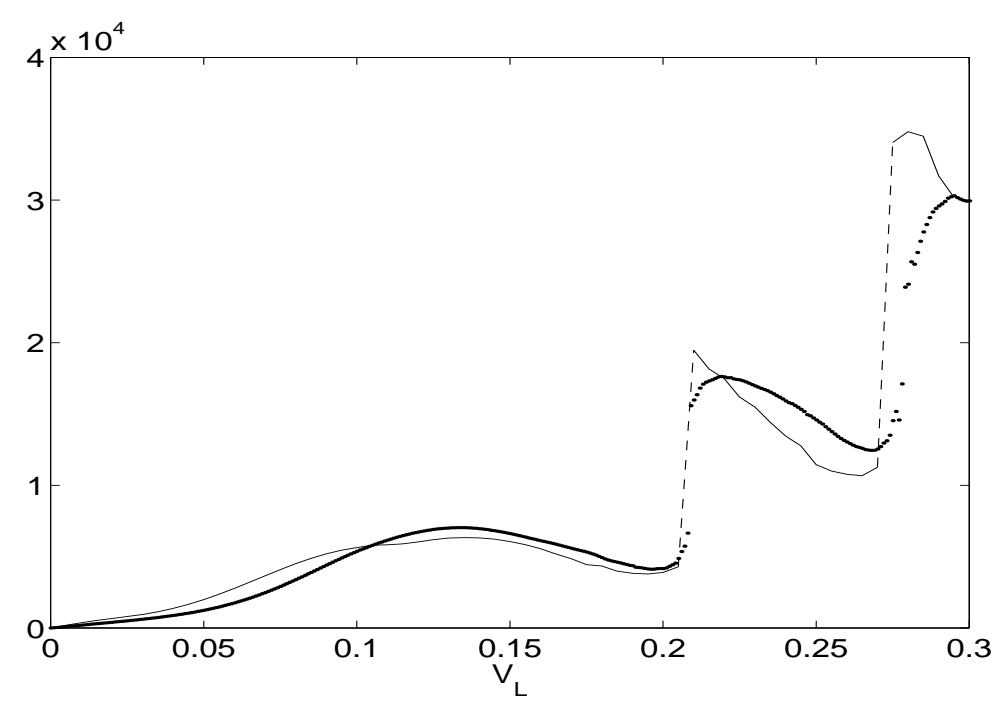

Figure 14: Effective current density (in $\mathrm{A} / \mathrm{cm}^{-2}$ ) versus applied voltage (in $\mathrm{V}$ ) for a resonant tunneling diode with two viscosity constants: $\nu=\nu_{0}$ (thin solid line) and $\nu=2 \nu_{0}$ (thick dotted line) with $\nu_{0}=0.00289$.

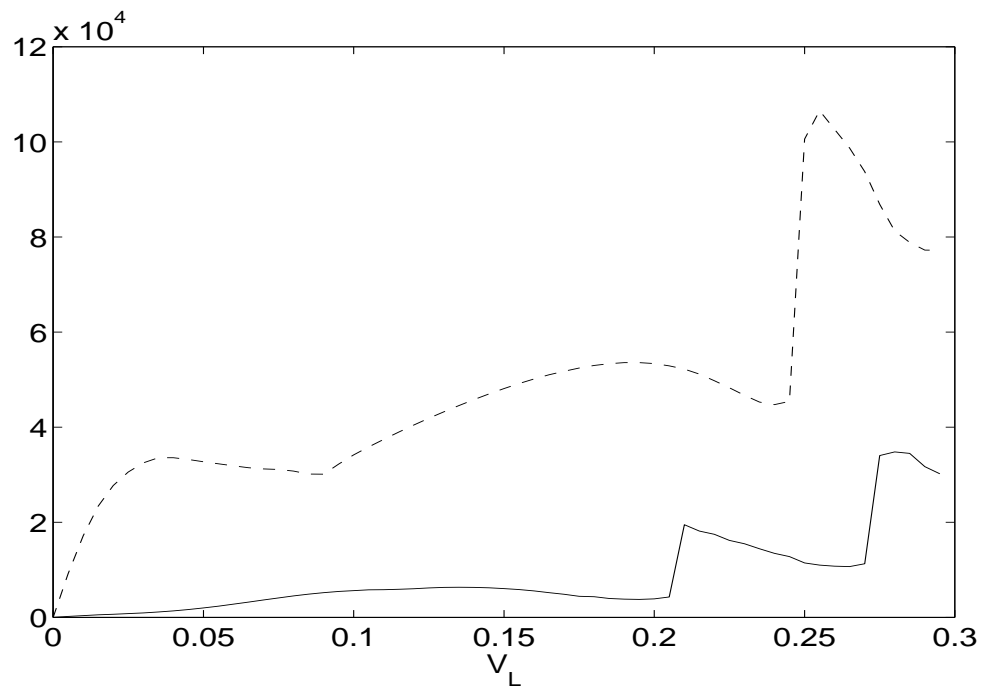

Figure 15: Effective current density (in $\mathrm{A} / \mathrm{cm}^{-2}$ ) versus applied voltage (in $\mathrm{V}$ ) for a resonant tunneling diode with lattice temperature $T_{0}=77 \mathrm{~K}$ (solid line) and $T_{0}=300 \mathrm{~K}$ (dashed line). 


\section{References}

[1] M. Ancona. Diffusion-drift modeling of strong inversion layers. COMPEL 6 (1987), 11-18.

[2] D. Aregba-Driollet and R. Natalini. Discrete kinetic schemes for multidimensional systems of conservation laws. SIAM J. Numer. Anal. 37 (2000), 1973-2004.

[3] P. Argyres. Quantum kinetic equations for electrons in high electric and phonon fields. Phys. Lett. A 171 (1992), 373-379.

[4] A. Arnold, J.L. López, P. Markowich, and J. Soler. An analysis of quantum Fokker-Planck models: a Wigner function approach. To appear in Rev. Mat. Iberoam., 2004.

[5] U. Ascher, P. Markowich, P. Pietra, and C. Schmeiser. A phase plane analysis of transonic solutions for the hydrodynamic semiconductor model. Math. Models Meth. Appl. Sci. 1 (1991), 347-376.

[6] F. Buot and K. Jensen. Lattice Weyl-Wigner formulation of exact many-body quantumtransport theory and applications to novel solid-state quantum-based devices. Phys. Rev. B 42 (1990), 9429-9457.

[7] A. Caldeira and A. Leggett. Path integral approach to quantum Brownian motion. Physica A 121A (1983), 587-616.

[8] F. Castella, L. Erdös, F. Frommlet, and P. Markowich. Fokker-Planck equations as scaling limits of reversible quantum systems. J. Stat. Phys. 100 (2000), 543-601.

[9] P. Caussignac, J. Descloux, and A. Yamnahakki. Simulation of some quantum models for semiconductors. Math. Models Meth. Appl. Sci. 12 (2002), 1049-1074.

[10] Z. Chen. A finite element method for the quantum hydrodynamic model for semiconductor devices. Computers Math. Appl. 31 (1996), 17-26.

[11] Z. Chen, B. Cockburn, C.L. Gardner, and J.W. Jerome. Quantum hydrodynamic simulation of hysteresis in the resonant tunneling diode. J. Comp. Phys. 117 (1995), 274-280.

[12] Z. Chen, B. Cockburn, J.W. Jerome, and C.-W. Shu. Mixed-RKDG finite element methods for the 2-D hydrodynamic model for semiconductor device simulations. VLSI Design 3 (1995), $145-158$.

[13] P. Degond, F. Méhats, and C. Ringhofer. Quantum energy-transport and drift-diffusion models. Preprint, Université Paul Sabatier, Toulouse, France, 2003.

[14] P. Degond and C. Ringhofer. Quantum moment hydrodynamics and the entropy principle. J. Stat. Phys. 112 (2003), 587-628.

[15] L. Diósi. On high-temperature Markovian equation for quantum Brownian motion. Europhys. Lett. 22 (1993), 1-3.

[16] B. C. Eu and K. Mao. Quantum kinetic theory of irreversible thermodynamics: low-density gases. Phys. Rev. E 50 (1994), 4380-4398. 
[17] F. Frommlet, P. Markowich, and C. Ringhofer. A Wigner function approach to phonon scattering. VLSI Design 9 (1999), 339-350.

[18] I.M. Gamba and A. Jüngel. Positive solutions of singular equations of second and third order for quantum fluids. Arch. Rat. Mech. Anal. 156 (2001), 183-203.

[19] C.L. Gardner. Numerical simulation of a steady-state electron shock wave in a submicron semiconductor device. IEEE Trans. Electr. Dev. 38 (1991), 392-398.

[20] C.L. Gardner. The quantum hydrodynamic model for semiconductor devices. SIAM J. Appl. Math. 54 (1994), 409-427.

[21] C.L. Gardner. Resonant tunneling in the quantum hydrodynamic model. VLSI Design 3 (1995), 201-210.

[22] C.L. Gardner and C. Ringhofer. Smooth quantum potential for the hydrodynamic model. Phys. Rev. E 53 (1996), 157-167.

[23] C.L. Gardner and C. Ringhofer. Numerical simulation of the smooth quantum hydrodynamic model for semiconductor devices. Comp. Meth. Appl. Mech. Engin. 181 (2000), 393-401.

[24] I. Gasser, P. Markowich, and C. Ringhofer. Closure conditions for classical and quantum moment hierarchies in the small-temperature limit. Transp. Theory Stat. Phys. 25 (1996), 409-423.

[25] I. Gasser, P. Markowich, D. Schmidt, and A. Unterreiter. Macroscopic theory of Charged quantum fluids. In P. Marcati et al. (eds.) Mathematical Problems in Semiconductor Physics. Research Notes in Mathematics Series 340, Pitman, 1995, 42-75.

[26] M.P. Gualdani and A. Jüngel. Analysis of the viscous quantum hydrodynamic equations for semiconductors. Submitted for publication, 2003.

[27] P.M. Gualdani, A. Jüngel, and G. Toscani. Exponential decay in time of solutions of the viscous quantum hydrodynamic equations. Appl. Math. Lett. 16 (2003), 1273-1278.

[28] M. T. Gyi and A. Jüngel. A quantum regularization of the one-dimensional hydrodynamic model for semiconductors. Adv. Diff. Eqs. 5 (2000), 773-800.

[29] S. Jin and Z.P. Xin. The relaxation schemes for systems of conservation laws in arbitrary space dimension. Commun. Pure Appl. Math. 48 (1995), 235-278.

[30] A. Jüngel. A steady-state potential flow Euler-Poisson system for charged quantum fluids. Comm. Math. Phys. 194 (1998), 463-479.

[31] A. Jüngel. Quasi-hydrodynamic Semiconductor Equations. Birkhäuser, Basel, 2001.

[32] A. Jüngel and H. Li. Quantum Euler-Poisson systems: global existence and exponential decay. To appear in Quart. Appl. Math., 2004.

[33] A. Jüngel, M.C. Mariani, and D. Rial. Local existence of solutions to the transient quantum hydrodynamic equations. Math. Models Meth. Appl. Sci. 12 (2002), 485-495. 
[34] A. Jüngel and R. Pinnau. A positivity-preserving numerical scheme for a nonlinear fourthorder parabolic equation. SIAM J. Num. Anal. 39 (2001), 385-406.

[35] A. Jüngel and S. Tang. A relaxation scheme for hydrodynamic equations for semiconductors. Appl. Numer. Math. 43 (2002), 229-252.

[36] E. Madelung. Quantentheorie in hydrodynamischer Form. Z. Physik 40 (1927), 322-326.

[37] P. Markowich, C. Ringhofer, and C. Schmeiser. Semiconductor Equations. Springer, Vienna, 1990.

[38] P. Pietra and C. Pohl. Weak limits of the quantum hydrodynamic model. VLSI Design 9 (1999), 427-434.

[39] R. Pinnau and A. Unterreiter. The stationary current-voltage characteristics of the quantum drift-diffusion model. SIAM J. Num. Anal. 37 (1999), 211-245.

[40] C.-W. Shu. Essentially non-oscillatory and weighted essentially non-oscillatory schemes for hyperbolic conservation laws. ICASE Report No. 97-65, NASA Langley Reserach Center, Hampton, USA, 1997.

[41] M. Taylor. Pseudodifferential Operators. Princeton University Press, Princeton, 1981.

[42] E. Wigner. On the quantum correction for thermodynamic equilibrium. Phys. Rev. 40 (1932), 749-759.

[43] B. Zhang and J.W. Jerome. On a steady-state quantum hydrodynamic model for semiconductors. Nonlin. Anal. 26 (1996), 845-856. 